\title{
Benchtop autoclaves: Are you looking after your water reservoirs properly?
}

\author{
A preliminary investigation of the microbiology and endotoxin content in the water reservoirs of benchtop non-vacuum \\ autoclaves by M. V. Martin and Y. Dailey Br Dent J 2001; 191: 622-624
}

\section{Summary}

To determine the microbial content and endotoxin concentration in the water reservoirs of benchtop autoclaves used in general dental practice.

\section{Design}

The study was done in two stages. Firstly water samples were taken daily from the reservoirs of 20 autoclaves used in general dental practices for six days. The microbial content and endotoxin concentration was determined. Second the practitioners were instructed to wash and clean the reservoirs of the autoclaves each morning prior to refilling with fresh water. The reservoirs were sampled in the evenings after a day's use and the microbial content and endotoxin concentration determined.

\section{Results}

The total viable count of bacteria before cleaning varied from $2300-8 \times 10^{4} \mathrm{cfu} / \mathrm{ml}$ and after from $0-40 \mathrm{cfu} / \mathrm{ml}$. The endotoxin concentrations before cleaning varied from $360-2200 \mathrm{EU} / \mathrm{ml}$ and after 0-80 EU/ml.

\section{Conclusions}

The reservoirs of non-vacuum benchtop autoclaves can become severely contaminated with micro-organisms, particularly Gramnegative bacteria. The endotoxin content of the reservoirs can get to high and unacceptable concentrations. Draining the water reservoir and cleaning on a daily basis reduces the microbial content and the endotoxin concentration to acceptable levels.

\footnotetext{
In brief

- The water reservoirs of a non-vacuum can contain a significant number of Gram-negative bacteria

- The reservoirs may also contain high concentrations of endotoxin

- The regular cleaning of water reservoirs is essential

- The water in reservoirs needs replacing frequently

- When considering purchasing an autoclave an accessible reservoir is essential
}

\section{Comment}

$\mathrm{D}$ econtamination of surgical instruments, including those used in dentistry, has achieved a heightened profile following the emergence of prion diseases. Safe and effective use of autoclaves is an important part of the decontamination process. In the past, there have been a number of reported adverse incidents involving contamination of reservoir water in bench top steam sterilisers. These have included corrosion of the chamber and foaming of water caused by contamination with a cleaner used in an ultrasonic bath. It is believed that such contamination of the reservoir water can be caused by using tap water, failing to rinse instruments properly after cleaning or failing to renew the water frequently.

This paper by Martin and Dailey focuses on the problem of bacterial contamination of water in autoclave reservoirs. Their results demonstrate high levels of Gram negative bacteria and significant amounts of endotoxin in reservoirs of 20 autoclaves in use in general dental practice. This is not a new problem. Indeed, the current guidelines from the British Dental Association (Advice Sheet A12) indicate that residual water should be drained from the autoclave chamber at the end of each day and the chamber should then be cleaned and dried. The data in Martin and Dailey's paper indicate clearly the effectiveness of this procedure in reducing contamination of reservoir water.

There has been further debate over the quality of water for use in autoclave reservoirs. The Medical Devices Agency recommends 'Sterile Water for Injection BP', but states that 'Sterile Water for Irrigation' may be a suitable alternative. This is clearly very expensive and the BDA guidelines indicate that distilled or de-ionised water may be satisfactory.

Regardless of the type of water used, it is essential that it be changed regularly. Whilst the investigators did not seek to determine how frequently this was undertaken in each practice, the fact that biofilm had developed in all of the reservoirs suggests that they were not cleaned and dried on a daily basis.

The important message from this study is that adherence to a simple regime of draining and cleaning the autoclave reservoir nightly will result in a major reduction in bacterial contamination. Since none of the 20 practices had a policy on this process, it is probable that the procedure is often overlooked.

Recent studies have reported a lack of formal documentation for other infection control procedures in dental practice. There is likely to be a significant tightening of controls on decontamination procedures in all healthcare settings in the coming months. This paper is a timely reminder of the need for all practices to scrutinise their sterilisation and disinfection processes carefully in the light of current guidance.

\section{Jeremy Bagg}

Professor of Clinical Microbiology, University of Glasgow Dental School 